\title{
Influence of Nozzle Shape on the Performance of Low-Power Ar Plasma Jet
}

\author{
Alonso Hernan Ricci Castro, Konstantin Georgiev Kostov, and Vadym Prysiazhnyi
}

\begin{abstract}
The influence of nozzle geometry on the properties of atmospheric-pressure Ar plasma jet was investigated. Three different nozzle types were tested: 1) a tapered nozzle (to get a thin plasma plume); 2) a straight nozzle (used in most cases); and 3) an enlarged nozzle (to increase the active plasma area). The electrical properties and visual appearance of the generated plasma were studied with these three nozzles. The jet's performance for surface modification was evaluated by measuring the area of the samples with enhanced wettability and comparing it with the image analysis of the light emission over the sample surface. It has been concluded that limiting the nozzle diameter can be a solution to reduce the treatment area. However, the decrease in the treated area is limited due to the plasma spreading. The use of widen nozzle leads to increase in the treated area and possibility to control the mixture of the carrier gas with ambient air, making this nozzle configuration potentially interesting for other applications, as, for example, treatment of small 3-D objects.
\end{abstract}

Index Terms-Jet geometry, plasma jet, power measurements.

\section{INTRODUCTION}

I $\mathrm{N}$ the last years, atmospheric-pressure plasma jets (APPJs) have attracted much attention as tools for surface modifications suitable for many applications. The APPJs can generate a large variety of active species that can be useful for different industrial processes [1]-[3]. Due to their unique properties (such as local treatment and possibility to create room-temperature plasmas), the plasma jets are widely studied for medical purposes [4]-[6]. Different jet configurations were developed and examined intensively by many research groups. The plasma properties and their effect on the jet performance have been investigated, for example, plasma temperatures [7], electric properties [8], and qualitative [9] and quantitative [10] identification of active species.

Commonly used APPJ arrangement is a single (or double) electrode configuration (only $\mathrm{HV}$ electrode, or HV and grounded electrodes). Of course, the geometry and powering of the jets influence the efficiency of the plasma treatments and thus are one of the most often covered topics.

Manuscript received February 10, 2015; accepted July 17, 2015. Date of publication August 28, 2015; date of current version September 9, 2015. This work was supported in part by the Fundação de Amparo à Pesquisa do Estado de São Paulo under Grant 2013/06732-3 and in part by the Conselho Nacional de Desenvolvimento Científico e Tecnológico under Project 470995/2013-0.

The authors are with the Department of Physics and Chemistry, Faculty of Engineering of Guaratingueta, Sao Paulo State University, Guaratingueta 12516-410, Brazil (e-mail: alonso_elfisico@yahoo.com; kostov@feg.unesp.br; mr.vodik@gmail.com).

Color versions of one or more of the figures in this paper are available online at http://ieeexplore.ieee.org.

Digital Object Identifier 10.1109/TPS.2015.2463072
For example, several APPJ configurations were described in [11]-[13].

One of the issues often addressed in research papers is the dimensions of the plasma plume that is extracted from the jet nozzle. For some applications, especially for biomedical treatments, it is required to have a small area plasma plume with high concentration of active species [14]. On the other hand, industrial applications typically require a large treatment area.

The limited treatment area of APPJs led to the development of two concepts of enlarging the active plasma area: 1) multijet systems or 2) the treatment of the surface using sample manipulation (scanning). Both approaches have its advantages and disadvantages. In multijet systems, the individual plasma jets are interacting with each other not allowing easy scaling [15]. Scanning the surface with a single jet always leaves a question about homogeneity of treatment. The measurement of the treated area after the APPJ treatment was done, for example, in [16]. An Ar plasma jet extracted from a syringe tip and applied to a polymer surface affected a circular area of 8 -mm diameter. The influence of the gas flow dynamics (laminar, turbulent, or transition between those) on the plasma plume was reported in [17] and [18] using Schlieren photography. The influence of the substrate on the gas flow dynamics was demonstrated in [18] without the discussion about the plasma spreading over the surface and its influence for the material modifications. Important findings were presented in [19], where it was demonstrated that in turbulent mode, the streamer formation in Ar plasma is following the air diffusion.

The determination of plasma-treated area is especially crucial in several applications of tissue treatments [20]. For example, the plasma application can promote cell adhesion as was reported in [21]. In this case, it is interesting to enlarge the treatment area, as the treatment of large samples would dramatically decrease the local heating. Even in more common plasma applications like textile treatments, it is still a challenge to get faster treatment over a larger area to modify the surface properties and introduce favorable functionalities (see [22]).

In this paper, the influence of nozzle geometry on plasma generation, dissipated power, and wettability modifications of glass were studied. It will be demonstrated in the following that the nozzle geometry has a significant influence on the treatment area. In addition, the correlation between the plasma spreading over the surface (obtained by conventional camera) and the treated area assessed using the water spreading (unlike the contact angle, the amount of water droplet is much higher) is presented. 


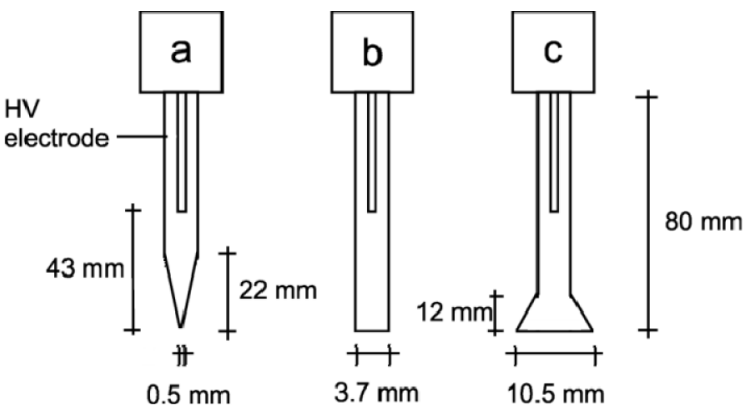

Fig. 1. Schematic of the three nozzle types. (a) Tapered nozzle. (b) Straight nozzle. (c) Horn-like nozzle.

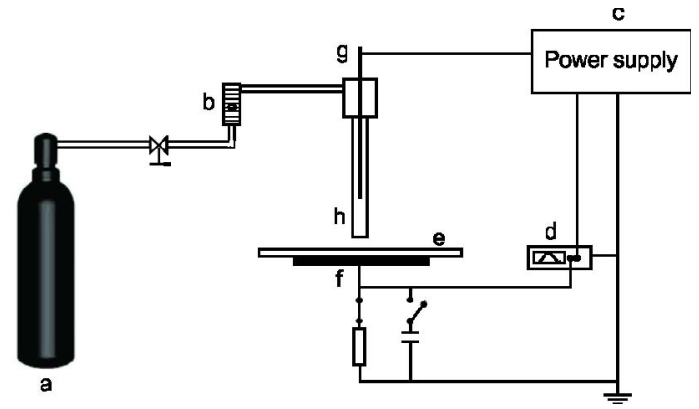

Fig. 2. Schematic of the experimental setup. (a) Argon container. (b) Gas flowmeter. (c) Power supply. (d) Oscilloscope. (e) Glass substrate. (f) Grounded electrode. (g) High-voltage electrode. (h) Plasma jet nozzle.

\section{EXPERIMENTAL DETAILS}

In this paper, three different plasma jet nozzle geometries were examined and their properties were compared. Fig. 1 shows a schematic of the nozzles that were used. All plasma jets were produced in Pyrex tubes with $7.3-\mathrm{mm}$ external diameter, 3.7- $\mathrm{mm}$ inner diameter, and $80-\mathrm{mm}$ length. The difference between them was the nozzle shape. The first one had a tapered pipette-shaped nozzle with a $0.5-\mathrm{mm}$ inner diameter at the end of the nozzle, which is shown in Fig. 2(a). The second one was just a perpendicularly cut Pyrex tube [the tube geometry was not changed, see Fig. 2(b)] that is commonly used in most laboratories devoted to the plasma jet research. The third one had widened horn-like nozzle geometry with 10.5-mm wide inner diameter at the end [Fig. 2(c)].

The plasma was generated using a Miniplus 4 power supply (GBS Elektronik, Germany). Its operation frequency ranges from 5 to $40 \mathrm{kHz}$, generating high ac voltage up to $40 \mathrm{kVp}-\mathrm{p}$. The Minipuls 4 consists of a low-voltage signal generator and a transformer cascade. The low-voltage signal generator was powered by a Voltcraft PS3620 laboratory power supply. The frequency of the generated HV signal was controlled by a Rigol DG1012 signal generator. A high voltage was supplied via a high-voltage electrode, a tungsten rod of 1.6-mm diameter with a square-shaped tip. The choice of squareshaped tip was done with a specific reason. We also tested the half-sphere and needle-shaped tips. It was noted that the generated filaments always emerged from one point of the tip (at the sphere or needle end). In some cases (for certain operation parameters), it influenced the homogeneity of the plasma inside the Pyrex tube. In addition, the filaments generated in this way propagate along the inner tube walls not covering the whole tube volume, thus leading to nonuniform tube heating (could led to an overheat issues after continuous operation of the jet) and potentially nonuniform surface treatments. When the square-shaped tip was used, the generated filaments are randomly distributed in the whole tube volume for all operation conditions.

The distance between the high-voltage electrode tip and the nozzle exit was fixed at $43 \mathrm{~mm}$. This value was also a result of geometry optimization. It was noted that for large electrodeto-nozzle distances, the plasma was not homogeneously spread over the tube volume as well as higher gas flows were necessary to extract the plasma. When the electrode-to-nozzle distance was decreased, the generated plasma appeared strongly nonuniform under many operation conditions, forming a single filament that was spiraled along the tube wall leading to a strong nonuniformity of the extracted plasma plume.

The plasma jets were operated in a flow of analytic argon (99.999\%). The gas flow was controlled by a Dwyer rotameter. In the current experiments, it was changed from 0.2 to $2 \mathrm{~L} / \mathrm{min}$. The plasma plume was directed on a glass substrate $(4 \mathrm{~cm} \times 4 \mathrm{~cm}$ area and $0.25-\mathrm{cm}$ thickness). It served as a contact surface for the further electrical characterization of the plasma jet. A grounded electrode, aluminum plate (3.7- $\mathrm{cm}$ diameter and $0.3-\mathrm{cm}$ thickness), was placed below the glass substrate. The experimental setup used in this paper is presented in Fig. 2.

The electrical characterization was done by measuring and analyzing the current and voltage waveforms. To calculate the dissipated electrical power that was transferred to the substrate, the Lissajous's figure method (or Q-V plot) was utilized. The applied voltage was measured by a voltage divider on the generator $(1: 2000)$. The discharge current was obtained from the voltage drop across a $47-\Omega$ inductance-free resistor. The transferred charge for the Q-V plot was measured from a 10-nF serial capacitor. The waveforms were recorded using Tektronix TDS3032 oscilloscope (300 MHz and $2.5 \mathrm{GS} / \mathrm{s}$ ).

The optical images of the plasma jets were collected using a common DSLR camera Nikon D3100 with Tamron 70-300 objective. Images of the plasma jets were taken without external lights with different exposition times ranging from 16 to $500 \mathrm{~ms}$.

\section{RESULTS}

\section{A. Visual Appearance of the Discharges}

Fig. 3 shows the typical view of APPJ with three nozzle geometries. Note that, visually plasma appeared diffusely spread in the tube, however, decreasing the exposition time showed that it consists of many short-living filaments that extended from the tip of metal electrode to the glass substrate. Due to the random spatial location of filaments for long exposition time, the plasma looks homogeneous. It has been noted that the gas flow plays a significant role on the filament propagation, as, for example, was also reported in [23]. In the mentioned reference authors demonstrated that the gas flow 


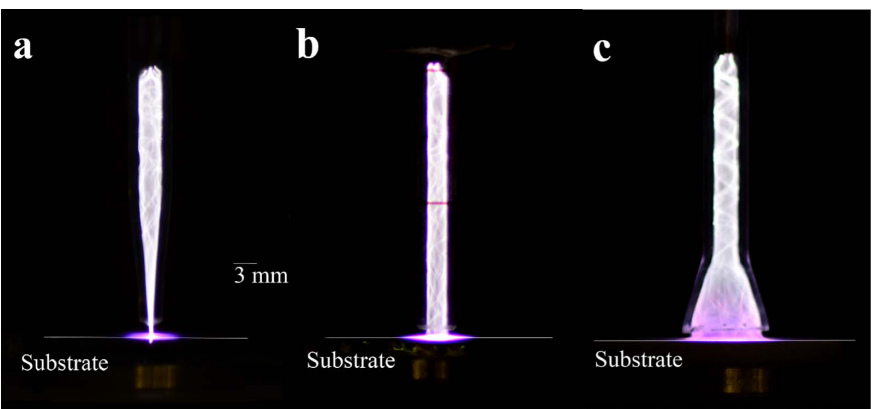

Fig. 3. Photographs of the plasma jet operated at an applied voltage of $15 \mathrm{kV}$ on a nozzle-to-substrate distance of $2 \mathrm{~mm}$ and an exposition time of $33 \mathrm{~ms}$. (a) Tapered nozzle jet with an Ar flow of $0.4 \mathrm{~L} / \mathrm{min}$. (b) Straight nozzle jet with an Ar flow of $0.6 \mathrm{~L} / \mathrm{min}$. (c) Horn-like nozzle jet with an Ar gas flow of $0.8 \mathrm{~L} / \mathrm{min}$.

influence not only visual appearance of the plasma but also its electrical properties. In this paper, it was decided to operate the plasma jets with the gas flow when the plasma uniformly spreads inside the dielectric tube (from 0.4 to $0.8 \mathrm{~L} / \mathrm{min}$ ).

The plasma jet using the tube with a tapered nozzle formed a thin and spatially stable plume of about $10-\mathrm{mm}$ length for the gas flow not higher than $0.4 \mathrm{~L} / \mathrm{min}$. When the gas flow exceeded $0.4 \mathrm{~L} / \mathrm{min}$, the plume length decreased due to a turbulent flow regime (plasma at the plume end had a spiral shape).

When the jet with a straight nozzle was operated, for low gas flows (less than $1 \mathrm{~L} / \mathrm{min}$ ), the filaments had a complex shape and were propagated along the inner walls of the Pyrex tube. For high flows $(1.6 \mathrm{~L} / \mathrm{min}$ and higher), the most filaments did not touch the Pyrex tube and had the shape of almost straight lines. The produced plasma was consisted of filaments randomly distributed in the whole tube volume. Depending on the operation conditions, one can distinguish them by a naked eye, but at other operation conditions, the plasma looked almost diffuse. Plasma plume had a shower-like shape with a diameter of plasma that is slightly larger than the inner diameter of the tube and about $18 \mathrm{~mm}$ in length. When the plasma jet operated with high flows $(1.6 \mathrm{~L} / \mathrm{min}$ or higher), the plasma plume was nonsymmetrical, which we consider to be an undesirable operation condition.

When the tube terminated with the horn-like nozzle, the plasma looked similar to the one of straight tube. For this nozzle type and without the presence of a substrate, the plasma plume was extracted only in the case of high gas flows [more than $1.6 \mathrm{~L} / \mathrm{min}$, see Fig. 4(a)]. However, in this case, the plasma plume also had a strongly nonsymmetric shape (it was stick to a certain position of the Pyrex tube border). To get a uniform plasma spread over the substrate, it was necessary to decrease the nozzle-to-substrate distance to $3 \mathrm{~mm}$ or less [Fig. 3(c)]. In this case, it was possible to operate the plasma with lower gas flows (but not less than $0.6 \mathrm{~L} / \mathrm{min}$ ). If the distance between the nozzle and the substrate was less than $1 \mathrm{~mm}$, than the plasma formed inside the conical horn was mostly from argon gas and no air admixture was occurring inside the tube [the plasma had a typical white color, see Fig. 4(c)], but increasing the distance for more than $1 \mathrm{~mm}$

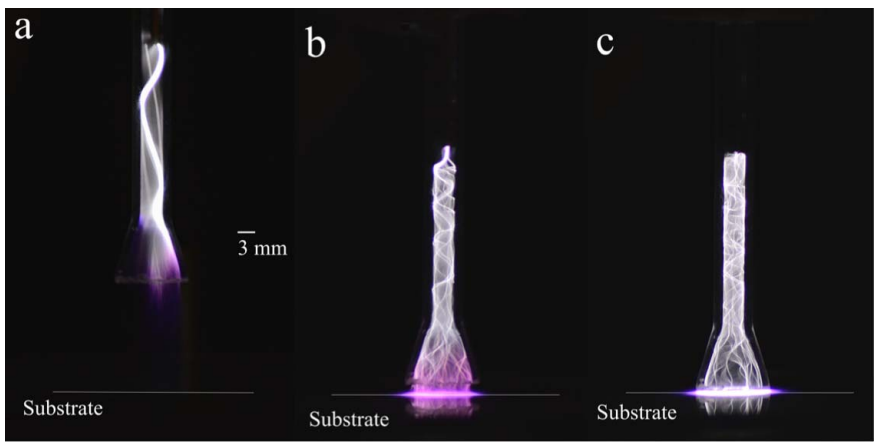

Fig. 4. Different snapshots showing the appearance of plasma jet with the horn-like nozzle taken at an exposition time of $25 \mathrm{~ms}$. (a) Nozzle-to-substrate distance of $16 \mathrm{~mm}$ and a gas flow of $1.6 \mathrm{~L} / \mathrm{min}$. (b) Nozzle-to-substrate distance of $3 \mathrm{~mm}$ and a gas flow of $0.8 \mathrm{~L} / \mathrm{min}$. (c) Nozzle-to-substrate distance of $0.5 \mathrm{~mm}$ and a gas flow of $0.8 \mathrm{~L} / \mathrm{min}$.
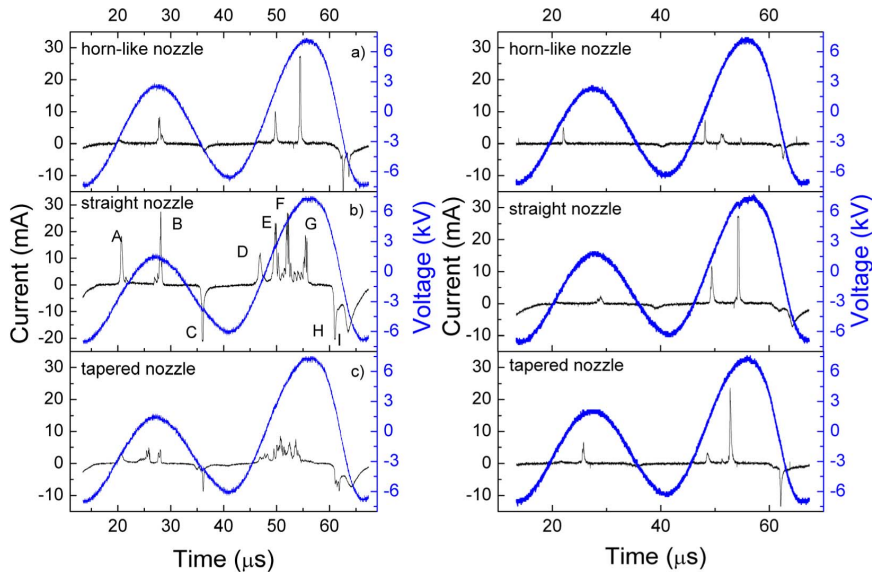

Fig. 5. Discharge current of the three plasma jets at (a) 2- and (b) 10-mm nozzle-to-substrate distance, Ar gas flow $(0.6 \mathrm{~L} / \mathrm{min}$, frequency of $19 \mathrm{kHz}$, and an voltage of $15 \mathrm{kVp}-\mathrm{p}$ ).

and up to $3 \mathrm{~mm}$ allowed the penetration of outside air into the tube, leading to the color change of plasma emission from white to types of purple [nitrogen emission of $\mathrm{N}_{2}(\mathrm{C}-\mathrm{B})$ band]. This was not observed in the jet with other two nozzles and opens a way how to control the plasma chemistry.

\section{B. Comparative Electric Characterization}

The electrical characterization properties of the plasma jets with three different types of plasma plume extraction were done for similar operation conditions: 1) an applied voltage of $15 \mathrm{kVp}-\mathrm{p}$; 2) an argon gas flow of $0.6 \mathrm{~L} / \mathrm{min}$; and 3) for two nozzle-to-substrate distances of $2 \mathrm{~mm}$ (as jet with the horn-like nozzle has a uniform plasma) and $10 \mathrm{~mm}$ (normal distance for operation of other two jet types).

The discharge current waveforms were recorded and analyzed. It was observed that for 2-mm distance, the current peaks changed depending on the nozzle geometry. Fig. 5(a) shows the current waveforms for each plasma jet in the presence of the glass substrate. The current peaks appeared in a certain time window when the breakdown was possible. Depending on the nozzle type, the number of the peaks varied. If one will start the period $(52.6 \mu \mathrm{s})$ from the minimum 


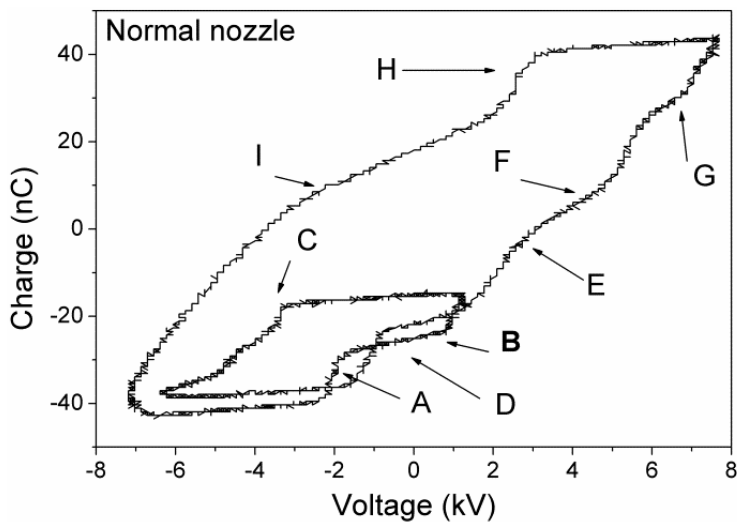

Fig. 6. Typical Q-V plot for the jet with the normal nozzle, a nozzle-tosubstrate distance of $2 \mathrm{~mm}$, an applied voltage of $15 \mathrm{kVp}-\mathrm{p}$, and a gas flow of $0.6 \mathrm{~L} / \mathrm{min}$.

position of the smaller voltage cycle (about $14 \mu$ s in Fig. 5), the current peaks would always appear in the time ranging from 6 to $14 \mu \mathrm{s}$ for the small voltage cycle and from 32 to $42 \mu \mathrm{s}$ for the higher voltage cycle. The amplitude of the current peaks was in the range from 5 to $30 \mathrm{~mA}$. The amplitudes of the current peaks could be correlated with the values of the dissipated power. When the distance nozzle substrate was $10 \mathrm{~mm}$, it was observed that the current waveform of the three jets looked same to the current waveform of the horn-like nozzle jet at 2-mm nozzle-to-substrate distance [see Fig. 5(a)].

When a capacitor was connected in series with the plasma jet, it was possible to calculate the dissipated power of the plasma using the area of the closed-loop figure that is built in coordinates voltage on the plasma source versus charge on the capacitor over one period. Because one period of the HV signal consisted of two sine-like cycles of different amplitudes, the Q-V plot had a shape of two loops (unlike one loop for the case of sine signal). To calculate the power, it is necessary to find the area of each loop separately and add them. When the snapshot of a single voltage period was taken together with the current waveform, it was observed that each shape change on the Lissajous figures can be related to a single current peak. As, for example, it is presented in Fig. 6, where the presented results were measured for the jet with the normal nozzle and the corresponding current waveform is presented in Fig. 4(b). As the plasma is unstable (constituted by random filaments), it is necessary to use averaging over many periods to obtain a mean value of the dissipated power. In this paper, the averaging of at least 500 consequent periods was used to calculate the dissipated power. In this case, it was possible to obtain the calculated power values within $3 \%$ error.

The variation of the dissipated power as a function of the nozzle-to-substrate distance is presented in Fig. 7. For distances more than $30 \mathrm{~mm}$, all jets did not have a contact with the substrate and the capacitor was charged due to the induced charges. In this case, the measured values are the same for all jets and the power is only slightly dependent on the distance. The differences in the mean power started to appear when the plasma plume approached the substrate. For the jet with the normal nozzle, it happened for nozzle-to-substrate distance between 20 and $30 \mathrm{~mm}$. The slope of the curve for the tapered nozzle jet started rising for

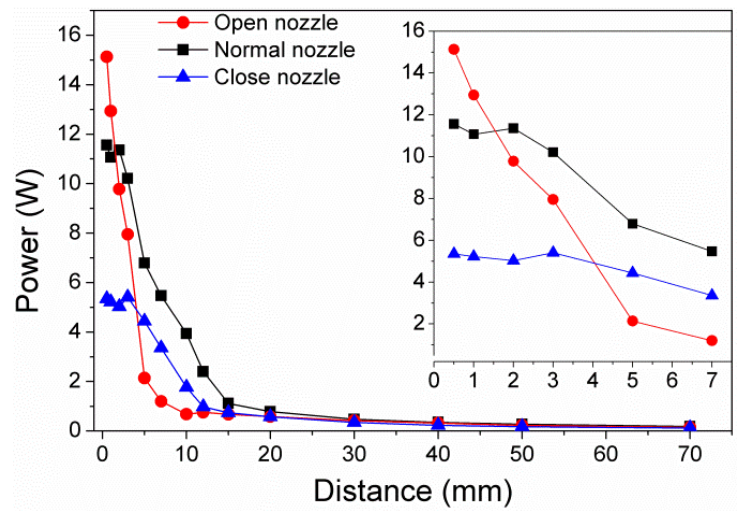

Fig. 7. Dissipated power for the three jets depending on the distance.
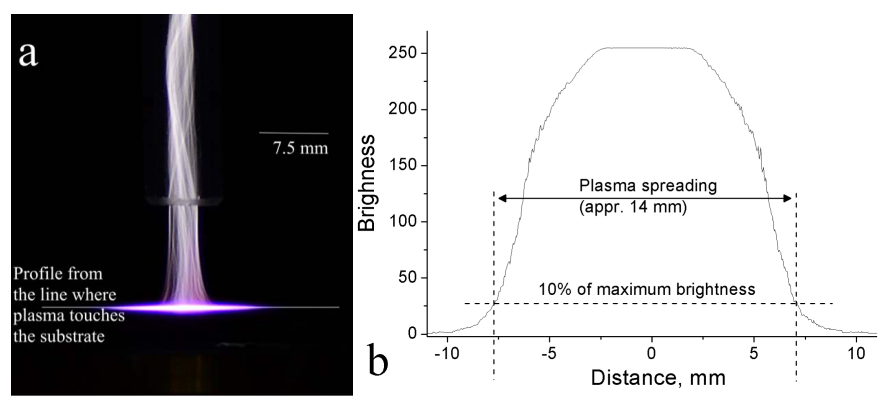

Fig. 8. (a) Photo and (b) light intensity profile for a normal nozzle (distance between jet and the substrate was $7.5 \mathrm{~mm}$ ).

distances starting from about $14 \mathrm{~mm}$. For the open nozzle jet, it appeared for distances starting from less than $10 \mathrm{~mm}$. For very short distances, the value of the dissipated power for the open nozzle jet overpassed the value of the power for the closed nozzle jet. It is due to the simple difference in the volume of the generated plasma. At short distances, the values of the dissipated power for both tapered and straight nozzles reached the saturation, which is probably due to gas dynamic effects.

\section{Plasma Spreading}

For short distances, the plasma plume spread on the glass substrate producing a circular light emission. The diameter of the plasma was evaluated from perpendicularly taken photos through brightness analysis using the ImageJ software. The time of exposition for all photos was fixed to $50 \mathrm{~ms}$ and $10 \%$ of the maximum intensity was considered to be the end of the light emission. Fig. 8 shows the example of the plasma spreading evaluation using the intensity profiles. Fig. 8(a) shows where the intensity profile was obtained and Fig. 8(b) shows a typical light intensity profile. Table I shows the values of the plasma spreading. As it was expected, the tapered nozzle produced the smallest plasma spreading over the glass substrate. Similar to the dependence of the dissipated power, at very short distances, the area with the light emission also decreased.

\section{Area of Improved Wettability}

The determination of treated area after the plasma treatments was done by dropping a deionized water of $32-\mu \mathrm{L}$ 
TABLE I

Plasma Spreading as a Function of the Nozzle Type AND NOZZLE-TO-SUbSTRATE DistanCE

\begin{tabular}{|c|c|c|c|c|c|}
\hline \multirow{2}{*}{ Nozzle type } & \multicolumn{5}{|c|}{ Distance $(\mathrm{mm})$} \\
\cline { 2 - 6 } & $0.5 \mathrm{~mm}$ & $1 \mathrm{~mm}$ & $1.5 \mathrm{~mm}$ & $2 \mathrm{~mm}$ & $10 \mathrm{~mm}$ \\
\hline Close nozzle & $11.6 \pm 0.3$ & $12.1 \pm 0.2$ & $13 \pm 0.3$ & $10.3 \pm 0.5$ & $6.4 \pm 0.3$ \\
\hline Normal nozzle & $22.3 \pm 0.5$ & $20.8 \pm 0.6$ & $20.2 \pm 0.6$ & $18.4 \pm 0.5$ & $11.9 \pm 0.5$ \\
\hline Open nozzle & $22.2 \pm 0.3$ & $22.1 \pm 0.2$ & $21 \pm 0.4$ & $18.5 \pm 0.4$ & - \\
\hline
\end{tabular}

TABLE II

Diameter of The AREa With High Wettability After the 3-s Plasma Jet Treatments

\begin{tabular}{|c|c|c|c|c|c|}
\hline \multirow{2}{*}{ Nozzle type } & \multicolumn{5}{|c|}{ Distance $(\mathrm{mm})$} \\
\cline { 2 - 6 } & $0.5 \mathrm{~mm}$ & $1 \mathrm{~mm}$ & $1.5 \mathrm{~mm}$ & $2 \mathrm{~mm}$ & $10 \mathrm{~mm}$ \\
\hline Close nozzle & $16.7 \pm 0.4$ & $16.2 \pm 0.8$ & $14.3 \pm 0.3$ & $14.2 \pm 0.3$ & $12.7 \pm 0.3$ \\
\hline Normal nozzle & $24.4 \pm 0.5$ & $23.1 \pm 0.2$ & $22.8 \pm 0.3$ & $22.1 \pm 0.4$ & $18.3 \pm 0.3$ \\
\hline Open nozzle & $28.4 \pm 0.4$ & $26.2 \pm 0.3$ & $25.1 \pm 0.2$ & $24.5 \pm 0.5$ & $10.2 \pm 0.9$ \\
\hline
\end{tabular}

volume on the glass surface. The reason why a common way to measure the contact angle of relatively small droplets with volumes up to few microliters was not chosen is that after a plasma treatment, a droplet of water formed a nonuniform noncircular shape, giving high error in contact angle determination. The treatment time for all conditions was fixed to $3 \mathrm{~s}$. When the drop was placed on the untreated glass surface, the drop formed a hemisphere meniscus with a diameter of about $7.8 \pm 0.4 \mathrm{~mm}$ and a contact angle about $60^{\circ}$. Table II shows the result of the wettability experiments. It is apparent that the nozzle shape has a significant influence on the treated area. However, it is important to note that the tapered nozzle (whose inner diameter is 20 times smaller than the hornlike nozzle) produced less than twice the smaller area of improved wettability. It is important to note that, even at the nozzle-to-substrate distance of $10 \mathrm{~mm}$, when no direct contact between the horn-like nozzle jet and the substrate was observed, the area with improved wettability was still larger than the diameter of the water drop on the untreated sample. It is due to a transport of the active species to the substrate.

\section{DISCUSSION}

The obtained results demonstrated that operating the jets at same conditions of high voltage, gas flow, and distance jet-tosample, it is possible to control the value of the jet power and the size of the area with high wettability. However, the light emission coming from the plasma spreading over the surface can be used only as a very rough estimation of the area that is affected by the plasma. The obtained results showed that the affected area is larger than the one visually in contact with the plasma. It can be easily explained as the long-living active species reach the substrate even outside the active plasma region.

It is important to note that the jet power density for all three jets had similar values. The power density can be easily estimated using the area obtained by the light emission. For the straight nozzle, the power density was about $0.8 \mathrm{~W} / \mathrm{cm}^{2}$, for the horn-like nozzle jet, the power density is about $0.9 \mathrm{~W} / \mathrm{cm}^{2}$, while for the tapered nozzle jet, the power density is about
$1.2 \mathrm{~W} / \mathrm{cm}^{2}$ when the nozzle-to-substrate distance. It is an important observation, especially for the jet with the hornlike nozzle that increasing the nozzle area, the jet does not lose the power density.

The obtained treatment area size of the tapered jet was somehow not expected. The almost 20 -fold decrease in the nozzle exit led to only about $60 \%$ decrease in the treatment area. The main reason of this drastic difference is the plasma spreading over the substrate.

The last issue to note is the possibility of the horn-like nozzle to control the plasma chemistry over the treated surface. It has been reported in the literature that the influx of ambient air strongly influences the chemistry of the plasma processing [24], [25]. In this respect, relative humidity presents a hard to control parameter that limits the applications of the plasma jets. Using the widen nozzle geometry, it was possible to create a virtually closed volume constantly purged with the carrier gas, thus having a low content of the ambient air in the active plasma region. It might be especially interesting when not Ar, but a combination of gases is used to achieve specific active species in the discharge area.

\section{CONCLUSION}

The influences of nozzle geometry on the jet visual appearance and electric characteristics, as well as plasma spreading and surface modifications were studied. A commonly used dielectric barrier discharge configuration of the plasma jet was used. It has been shown that the nozzle geometry has a strong influence on the contact area between the plasma and the substrate. The decrease in the nozzle diameter (20 times) led to a decrease in the treated area, but the observed decrease was only $60 \%$ due to a plasma spreading effect. The use of the horn-like nozzle led to the increase in the treated area and, when the nozzle-to-substrate distance is less than $1 \mathrm{~mm}$, the admixture of the ambient air with Ar can be prevented, showing a potential to control the plasma chemistry. It is to be additionally noted that changing the nozzle shape does not significantly change the energy density of plasma per unit of surface. We believe that the horn-like nozzle geometry has a potential interest for treating small objects, but requires additional studies to be performed.

\section{REFERENCES}

[1] S. Keller, P. Rajasekaran, N. Bibinov, and P. Awakowicz, "Characterization of transient discharges under atmospheric-pressure conditions applying nitrogen photoemission and current measurements," J. Phys. D, Appl. Phys., vol. 45, no. 12, p. 125202, Mar. 2012.

[2] J. J. Shi, X. T. Deng, R. Hall, J. D. Punnett, and M. G. Kong, "Three modes in a radio frequency atmospheric pressure glow discharge," J. Appl. Phys., vol. 94, no. 10, p. 6303, Oct. 2003.

[3] M. C. Kim, S. H. Yang, J.-H. Boo, and J. G. Han, "Surface treatment of metals using an atmospheric pressure plasma jet and their surface characteristics," Surf. Coatings Technol., vols. 174-175, pp. 839-844, Sep./Oct. 2003.

[4] K.-D. Weltmann et al., "Antimicrobial treatment of heat sensitive products by miniaturized atmospheric pressure plasma jets (APPJs)," J. Phys. D, Appl. Phys., vol. 41, no. 19, p. 194008, Sep. 2008.

[5] G. Daeschlein, T. von Woedtke, E. Kindel, R. Brandenburg, K.-D. Weltmann, and M. Jünger, "Antibacterial activity of an atmospheric pressure plasma jet against relevant wound pathogens in vitro on a simulated wound environment," Plasma Processes Polym., vol. 7, nos. 3-4, pp. 224-230, Mar. 2010. 
[6] K.-D. Weltmann et al., "Atmospheric pressure plasma jet for medical therapy: Plasma parameters and risk estimation," Contrib. Plasma Phys. vol. 49 , no. 9, pp. 631-640, Nov. 2009.

[7] S. Hofmann, A. F. H. van Gessel, T. Verreycken, and P. Bruggeman, "Power dissipation, gas temperatures and electron densities of cold atmospheric pressure helium and argon RF plasma jets," Plasma Sour. Sci. Technol., vol. 20, no. 6, p. 065010, Nov. 2011.

[8] J. L. Walsh, F. Iza, N. B. Janson, V. J. Law, and M. G. Kong, "Three distinct modes in a cold atmospheric pressure plasma jet," J. Phys. D, Appl. Phys., vol. 43, no. 7, p. 075201, Feb. 2010.

[9] J. Janča, M. Klíma, P. Slavíček, and L. Zajíčková, "HF plasma pencil-New source for plasma surface processing," Surf. Coatings Technol., vols. 116-119, pp. 547-551, Sep. 1999.

[10] L. Li et al., "OH radicals distribution in an $\mathrm{Ar}-\mathrm{H}_{2} \mathrm{O}$ atmospheric plasma jet," Phys. Plasmas, vol. 20, no. 9, p. 093502, Sep. 2013

[11] Y. Hong, N. Lu, J. Pan, J. Li, Y. Wu, and K. F. Shang, "Characteristic study of cold atmospheric argon plasma jets with rod-tube/tube high voltage electrode," J. Electrostatics, vol. 71, no. 2, pp. 93-101, Apr. 2013

[12] S. Bornholdt, M. Wolter, and H. Kersten, "Characterization of an atmospheric pressure plasma jet for surface modification and thin film deposition," Eur. Phys. J. D, vol. 60, no. 3, pp. 653-660, Dec. 2010

[13] M. Laroussi and X. Lu, "Room-temperature atmospheric pressure plasma plume for biomedical applications," Appl. Phys. Lett., vol. 87 no. 11 , p. 113902 , Sep. 2005

[14] J. Lademann et al., "Risk assessment of the application of a plasma jet in dermatology," J. Biomed. Opt., vol. 14, no. 5, p. 054025, Sep./Oct. 2009.

[15] M. Ghasemi, P. Olszewski, J. W. Bradley, and J. L. Walsh, "Interaction of multiple plasma plumes in an atmospheric pressure plasma jet array," J. Phys. D, Appl. Phys., vol. 46, no. 5, p. 052001, Jan. 2013.

[16] C. B. Mello, K. G. Kostov, M. Machida, L. R. O. de Oliveira Hein, and K. A. de Campos, "Surface modification of polycarbonate by atmospheric-pressure plasma jets," IEEE Trans. Plasma Sci., vol. 40, no. 11 , pp. 2800-2805, Nov. 2012.

[17] J.-S. Oh, O. T. Olabanji, C. Hale, R. Mariani, K. Kontis, and J. W. Bradley, "Imaging gas and plasma interactions in the surfacechemical modification of polymers using micro-plasma jets," J. Phys. D, Appl. Phys., vol. 44, no. 15, p. 155206, Apr. 2011.
[18] E. Robert et al., "Rare gas flow structuration in plasma jet experiments," Plasma Sour. Sci. Technol., vol. 23, no. 1, p. 012003, Jan. 2014.

[19] S. Iseni, A. Schmidt-Bleker, J. Winter, K.-D. Weltmann, and S. Reuter, "Atmospheric pressure streamer follows the turbulent argon air boundary in a $\mathrm{MHz}$ argon plasma jet investigated by $\mathrm{OH}$-tracer PLIF spectroscopy," J. Phys. D, Appl. Phys., vol. 47, pp. 152001-1-152001-6, Mar. 2014.

[20] G. Fridman et al., "Comparison of direct and indirect effects of non-thermal atmospheric-pressure plasma on bacteria," Plasma Process Polym., vol. 4, no. 4, pp. 370-375, May 2003.

[21] S. Yonson, S. Coulombe, V. Léveillé, and R. L. Leask, "Cell treatment and surface functionalization using a miniature atmospheric pressure glow discharge plasma torch," J. Phys. D, Appl. Phys., vol. 39, no. 16, p. 3508 , Aug. 2006

[22] C. X. Wang and Y. P. Qiu, "Two sided modification of wool fabrics by atmospheric pressure plasma jet: Influence of processing parameters on plasma penetration," Surf. Coatings technol., vol. 201, no. 14, pp. 6273-6277, Apr. 2007.

[23] F. Liu et al., "Electrical characteristics of monofilaments in dielectric barrier discharge plasma jets at atmospheric pressure," EPL, vol. 97, no. 6, p. 65001, Mar. 2012.

[24] B. van Gessel, R. Brandenburg, and P. Bruggeman, "Electron properties and air mixing in radio frequency driven argon plasma jets at atmospheric pressure," Appl. Phys. Lett., vol. 103, no. 6, p. 064103, Aug. 2013.

[25] A. Schmidt-Bleker, J. Winter, S. Iseni, M. Dünnbier, K.-D. Weltmann, and S. Reuter, "Reactive species output of a plasma jet with a shielding gas device-Combination of FTIR absorption spectroscopy and gas phase modelling," J. Phys. D, Appl. Phys., vol. 47, no. 14, pp. 145201-1-145201-12, Mar. 2014

Authors' photographs and biographies not available at the time of publication. 